\title{
Respiratory System Findings Numeric Result in Standard Unit
}

National Cancer Institute

\section{Source}

National Cancer Institute. Respiratory System Findings Numeric Result in Standard Unit. NCI Thesaurus. Code C124018.

The numerical identifier of a respiratory system finding result in standard units. 